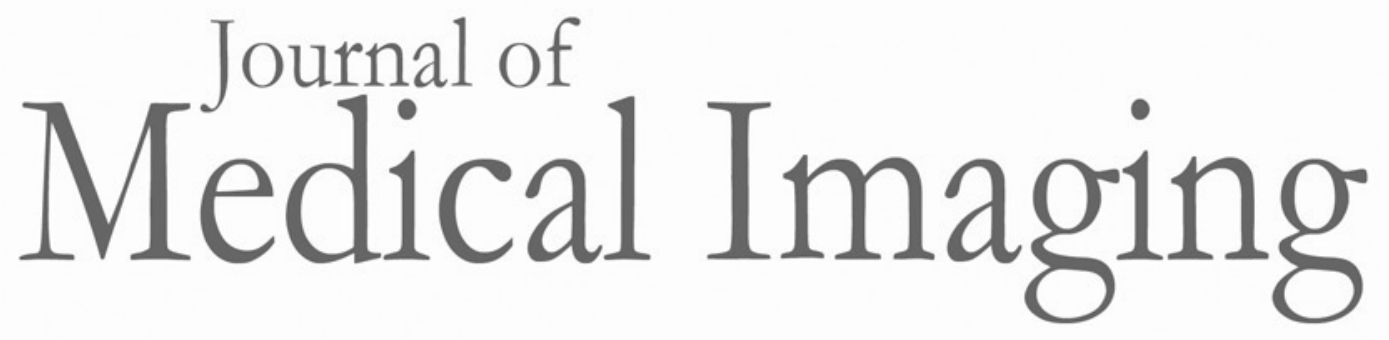

Medicallmaging.SPIEDigitalLibrary.org

\title{
MARIA M4: clinical evaluation of a prototype ultrawideband radar scanner for breast cancer detection
}

\author{
Alan W. Preece \\ Ian Craddock \\ Mike Shere \\ Lyn Jones \\ Helen L. Winton
}




\title{
MARIA M4: clinical evaluation of a prototype ultrawideband radar scanner for breast cancer detection
}

\author{
Alan W. Preece, ${ }^{a, *}$ lan Craddock, ${ }^{b}$ Mike Shere, ${ }^{c}$ Lyn Jones, ${ }^{c}$ and Helen L. Winton ${ }^{d}$ \\ ${ }^{a}$ Bristol University, School of Clinical Sciences, Southwell Street, Bristol BS2 8EJ, United Kingdom \\ ${ }^{b}$ Bristol University, School of Electrical and Electronic Engineering, Merchant Venturers Building, Woodland Road, Bristol BS8 1UB, \\ United Kingdom \\ 'North Bristol NHS Trust, Bristol Breast Care Centre, Southmead Hospital, Bristol BS10 5NB, United Kingdom \\ ${ }^{\mathrm{d}}$ Micrima Limited, The Engine Shed, Station Approach, Bristol BS1 6QH, United Kingdom
}

\begin{abstract}
A microwave imaging system has been developed as a clinical diagnostic tool operating in the 3 - to $8-\mathrm{GHz}$ region using multistatic data collection. A total of 86 patients recruited from a symptomatic breast care clinic were scanned with a prototype design. The resultant three-dimensional images have been compared "blind" with available ultrasound and mammogram images to determine the detection rate. Images show the location of the strongest signal, and this corresponded in both older and younger women, with sensitivity of $>74 \%$, which was found to be maintained in dense breasts. The pathway from clinical prototype to clinical evaluation is outlined. () The Authors. Published by SPIE under a Creative Commons Attribution 3.0 Unported License. Distribution or reproduction of this work in whole or in part requires full attribution of the original publication, including its DOI. [DOI: 10.1117/1.JMI.3.3.033502]
\end{abstract}

Keywords: breast cancer; MARIA; ultrawideband; multistatic radar; imaging; clinical evaluation.

Paper 16024R received Feb. 3, 2016; accepted for publication Jun. 30, 2016; published online Jul. 20, 2016.

\section{Introduction}

Breast cancer (BC) is the most frequently diagnosed cancer in women worldwide, with nearly 1.7 million new cases diagnosed in 2012, and more than half of BC cases and deaths occurring in economically developing countries. ${ }^{1-3}$ Asian countries, which represent $59 \%$ of the global population, have the largest burden of $\mathrm{BC}$, with $39 \%$ of new cases, followed by Europe at $28 \%$. 3,4 In 2012, deaths from BC in the USA accounted for 783,000 years of potential life lost and an average of 19 years of life lost per death..$^{5}$ Early detection has been shown to be associated with reduced $\mathrm{BC}$ morbidity and mortality ${ }^{6,7}$ and the goal of BC screening programs is to reduce both. Most BCs are detected due to clinical symptoms or by screening mammography (MMG). The standard way to assess suspicious lesions is with the so-called triple assessment: clinical examination, imaging by MMG and ultrasound (US), and image-guided needle biopsy. Magnetic resonance imaging is currently used for initial cancer detection in women at high risk of developing BC but is a complex investigation with high direct and indirect costs. ${ }^{8-11}$ MMG is one of the most effective detection techniques, but suffers from relatively low sensitivity, entails exposure to ionizing radiation and also involves uncomfortable compression of the breast. MMG also performs less well in younger, more dense breasts, which is pertinent as breast density is now established as an independent risk factor for developing BC irrespective of other known risk factors. ${ }^{12-16}$ This coupled with the increased risk from ionizing radiation in younger women, restricts the lower age for use based on risk/benefit ratio. Limitations of MMG have resulted in research into alternative methods for imaging of breasts with microwave detection of breast tumors

*Address all correspondence to: Alan W. Preece, E-mail: A.W.Preece@ bristol. ac.uk being a potential nonionizing alternative. ${ }^{17}$ Initial results of microwave radar-based imaging have been presented ${ }^{17-23}$ and approaches rely on a difference in the dielectric properties (Dk) of normal and malignant breast tissues. ${ }^{24-31}$ The breast as an organ is unique in the human body in that basic structure consists of glandular tissue (high dielectric constant, high conductivity, and radioopaque) in a fat (low dielectric constant, low conductivity, and relatively radiolucent)-based matrix. Inclusions, such as a tumor, are also of high permittivity, enhanced by the angiogenic increase in vascularity, and cysts contain fluid, which also have very high permittivity.

Some early measurements at $3.2 \mathrm{GHz}^{26}$ indicate that the most common relative permittivity values for breast fat were 4 to 4.5 , for normal glandular tissue 10 to 25 and for malignant tissues 45 to 60 , but overlaps occurred so that values up to 55 and down to 10 for normal and malignant tissues, respectively, occurred. Glandular tissue is distributed whereas malignant and cystic tissue tends at diagnosis to be discrete and, therefore, much easier to image. Similar results were obtained using completely different measurement techniques by Sugitani et al. ${ }^{32}$ showing overlap of tissue values.

Such inclusions alter the speed of propagation of radio waves passing through the tissue and the higher conductivity results in radio wave absorption. These changes mean that the phase and amplitude of a signal is affected by inclusions. In order to image inclusions, an array of antennas transmit signals in turn to be detected by all the other nontransmitting antennas-a so-called multistatic array. The choice of frequency for such a radar system is a compromise between absorption of radio waves (which increases with frequency) and resolution (which increases with decreasing wavelength). Availability of a suitable radio wave transmitter and receiver [in this case, a vector network analyzer (VNA)] is also a factor. An ultrawideband (UWB) signal from 3 to $8 \mathrm{GHz}$ is used in this development. 


\section{Methods and Materials}

A series of prototype MARIA radar scanners were constructed within the Electrical and Electronic Engineering Department of the University of Bristol with funding from Micrima Ltd. All systems were based on multistatic radar operation, originally proposed for land mine detection by Benjamin. ${ }^{33}$ Prototypes have evolved from an initial 16-antenna array ${ }^{34}$ through to a 31-element UWB slot antenna system (MARIA M3). ${ }^{35}$ To increase the number of antennas, arrays have been redesigned with new smaller UWB antennas. For improved imaging performance and reduced scanning times, a new 60-element antenna array system has been designed (MARIA M4). ${ }^{36}$ This system consists of 60 wide-slot antenna elements positioned in a hemispherical arrangement. ${ }^{36,37}$ The antennas operate over a frequency range of 3 to $8 \mathrm{GHz}$ in a cavity loaded slot arrangement. ${ }^{38}$ Each antenna is designed to couple into a dielectric constant environment of $\mathrm{Dk}=10$.

To interface the antenna into tissue and to provide a fixed spacer to place the imaged tissue volume in the antenna far field, a separate fixed coupling shell with a uniform $\mathrm{Dk}=10$ is employed between the antennas and the breast tissue. This shell leaves a space between the antenna face and the shell is filled with a water-/oil-based coupling fluid also with a $\mathrm{Dk}=10$.

The coupling shell and coupling fluid allows the antenna array not only to match the antenna into its surrounding environment and provide maximum radiated power, but as importantly, provides a method to allow the antenna array to rotate underneath the fixed shell. The system signal source is a VNA operating in the range of 3 to $8 \mathrm{GHz}$ employing standard stepped continuous wave mode. To couple the antennas to the VNA source/receiver, a low-loss high-isolation switch matrix allows a single signal to be connected to any one of the 60 antennas and groups of receiving antenna signals to be, simultaneously, received at the VNA

The system collects signal data from the finished array by serially energizing each antenna and collecting the scattering parameter values at each incident frequency from the receive signals collected at all remaining antennas. This method results in a set of signal data for each of the bistatic ray paths. Due to antenna reciprocity, we can reduce the number of bistatic signals collected to half of the $3540 / 2$ or 1770 . This reduces the overall scan time.

As signals are transmitted from each antenna, every signal passes through the external coupling shell and into the tissue volume. Multiple reflections occur within the antenna array and its associated coupling shell and coupling fluid and at the interface between the coupling shell and the breast skin surface. The breast skin surface has an estimated Dk of 25, so a significant portion of the incident signal is reflected (depending on its incident angle at this interface). Signals that penetrate into tissue are then reflected at random angles from the surfaces of tissue dielectric discontinuities within the tissue volume.

The various tissue types found in the breast have clearly identifiable dielectric constants in the microwave frequency range, ${ }^{27,32}$ which result in incident signals being reflected and attenuated differently at each interface between the tissue types. It is these signals the system collects and accumulates at each point in the estimated tissue space. These intratissue response signals are very small in comparison to the signals from the hardware/skin reflection signals that also appear in the final signal set; thus, a method to remove the nontissue generated signals from the final set is necessary before image generation.
Each complete image scan of the breast is a result of two separate scans offset from one another by a fixed angle. Unwanted signals produced by hardware and skin reflections are almost identical and appear at the same time position in each scan; therefore, they can be eliminated. In contrast, a tumor response will appear at different time positions in these two measured sets (except on the axis of rotation).

During image generation the single scans are subtracted from one another. This leaves the "nonstationary" signals intact and significantly reduces the "stationary" signals so that the signals generated by the tissue volume reflections predominate.

Image generation from the resulting RF signal data makes a number of assumptions. We assume that within the angle of array rotation (a) distance between antennas and skin remains unchanged, (b) skin properties and thickness are the same, (c) normal breast tissue properties do not change, and (d) a uniform dielectric constant of $\mathrm{Dk}=10$ exists with the hardware and breast tissue across all frequencies of interest. These assumptions allow an estimate to be made of the location of a received signal based on the time-of-flight to and from the target location and based on the transition time of signals at each frequency in a medium whose $\mathrm{Dk}=10$.

To generate the image, the system uses a modified version of the classical delay-and-sum (DAS) beamforming algorithm. 23,39,40 First, we perform the preprocessing steps, consisting of extraction of the tumor response from measured data, ${ }^{23}$ equalization of tissue losses, and then equalization of radial spread of the spherical wavefront. Next, appropriate time delays for all received signals are computed. The time delay for a given transmitting and receiving antenna is calculated based on the antenna's position, position of the focal point $r=(x, y, z)$, as well as an estimate of average wave propagation speed, which in our case is assumed to be constant across the band. During the focusing, the focal point moves from one position to another within the breast; at each location, all time-shifted responses are coherently summed and integrated. Integration is performed on the windowed signal, and the length of the integration window is chosen according to the system bandwidth, which is $50 \%$ longer than the synthetic pulse duration and was set to $0.55 \mathrm{~ns},{ }^{23}$ to form a three-dimensional (3-D) map of scattered energy. The main advantage of the DAS algorithm is its simplicity, robustness, and short computation time.

The 3-D map of spatial energy is presented to the user as a colored image comprising slices along three axes: craniocaudal (CC), mediolateral, and physician point-of-view. The energy image is normalized to the maximum energy value within the image. The image presented to the reader is thresholded (calibrated) at $70 \%$ of the maximum, which corresponds to the significant scatters within the breast as determined through extensive phantom experimental work. ${ }^{35}$ Typically energy values less than $70 \%$ of the maximum correspond to clutter. An isometric 3-D rotatable image is provided showing an iso-surface representation of the energy values whose relative contrast is adjustable by the image reader.

\subsection{Clinical Equipment}

The microwave components and supporting mechanical parts are incorporated into a fully integrated bed/system cabinet design (Fig. 1).The antenna array position is adjustable with the patient in position on the bed. It can be raised and lowered and is provided with lateral and cranial/caudal adjustment to allow the operator to optimally position the breast within the 
(a)

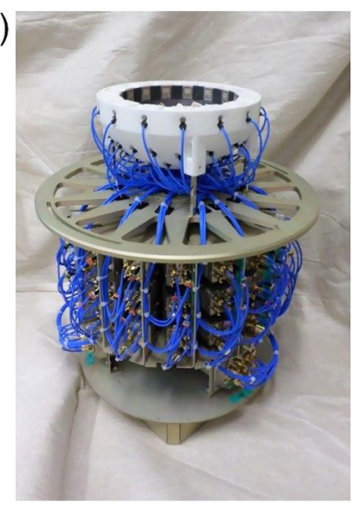

(b)

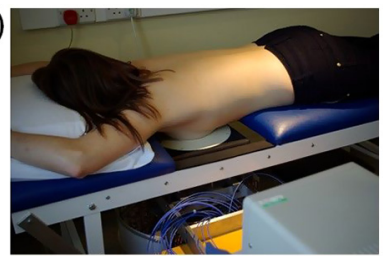

(c)

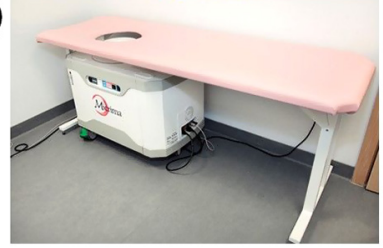

Fig. 1 MARIA M4/M5 array and bed system. (a) Latest design rotatable 60 antenna array and switch assembly which moves as a unit around the breast cup. (b) MARIA M4 in position in the clinic. (c) MARIA M5 operationally identical to M5 design but in a clinically more acceptable and integrated package as currently in use.

scanning cup in terms of fit without the patient having to move during normal clinical application. The system cabinet can also be rotated out from under the bed to allow introduction of additional inserts designed to accommodate smaller breast cup sizes into the basic breast cup [Fig. 1(c)].

\subsection{Patient Population}

The MARIA M4 prototype system was initially tested for efficacy in women attending symptomatic breast care clinics. Eighty-six patients were identified by clinicians as meeting study inclusion criteria [symptomatic clinic, to be examined by US and MMG, able to lie prone and having breast size within the range of available cups (310 to $850 \mathrm{ml}$ )] and after giving informed consent were recruited at either Frenchay or Southmead Hospital, Bristol, U.K., and included in the observational, prospective MARIA M4 clinical evaluation study [approved by Central and South Bristol Research Ethics Committee (REC) 06/Q2006/30]. The type of lesions included were mainly cysts and cancers but a small number of "other" conditions that had mammogram and US were included. These conditions were a mix of hematoma, lipoma, or fibroadenoma.

\subsection{Procedure in Clinic}

Patients had an US examination and MMG, and where possible a cytology or histology examination (if appropriate and for patient benefit) as part of normal clinical procedure. Patients were scanned using MARIA M4 prior to any surgical or biopsy intervention. Patients were required to lie prone with the breast

inserted into a ceramic cup lined with a small amount of "coupling fluid" of dielectric constant 10 and attenuation of $0.8 \mathrm{~dB} / \mathrm{cm}$ at $3 \mathrm{GHz}{ }^{41}$ The scan consisted of checks for goodness of fit of the breast inside the cup (lack of air gap), followed by at least two further scans of about $30 \mathrm{~s}$ each. Data was processed offline.

\subsection{Data Collection}

Data collected were Breast Imaging Reporting and Data System (BI-RADS) score, ${ }^{42}$ age, menopausal status, and breast size. Evaluation of MARIA M4 scans consisted of two stages: a judgment of lesion(s) type, size, and location using all available clinical data by a researcher who had no knowledge of the MARIA image, and an assessment of the MARIA image by an engineer who had no access to the clinical data or image. The two observations were then compared jointly by the two observers to decide on the available data of a good correspondence, failure to correspond, or a need to exclude. In this, the results from US with or without MMG were the "gold standard." Additionally, a nested evaluation was undertaken in which a blind read of all available MMGs was completed for MARIA M4 study patients $(n=66)$. All patients' identifiable information was removed from MMGs by a patient archiving communication system (PACS) administrator and a blind read of the MMG was conducted by an experienced radiologist. Blind read results were compared to the original clinical result using all available clinical information and to MARIA M4 detection (versus "gold standard" results).

\section{Results}

Of 86 MARIA M4 patients included in the study, a sensitivity score of 74\% (64/86) correspondence with the "gold standard" (mean age 51.4 years, age range 24 to 87, diagnoses: cysts $n=36(57 \%)$, cancer $n=20(31 \%)$, others $n=8(12 \%)$ was obtained (Table 1). Before reviewing a MARIA image, the location of the lesion within the breast was recorded on the basis of octant of breast, depth on US (allowing for degree of compression by the probe), and distance from the nipple as noted in clinical and imaging examinations. The sensitivity was judged by whether MARIA located an apparent lesion in the corresponding position, making subjective allowance for US probe compression and MMG breast compression. The MARIA image was produced by an engineer "blind" to the clinical status. On this basis there was $75 \%(45 / 60)$ sensitivity in pre/ peri-menopausal women and $73 \%(19 / 26)$ in postmenopausal women. An example of a MARIA M4 scan is given at $70 \%$ threshold within the image [Fig. 2(a)].

Of the initial 86 studies reviewed, 66 had a MMG available for comparison. Of these cases there was 74\% (49/66) sensitivity for MARIA M4 compared to MMG (Table 2). Sensitivity was

Table 1 Patient demographics, sensitivity scores and diagnoses for MARIA M4 detection (versus gold standard).

\begin{tabular}{lccccccc} 
& Cases $n=86$ & Sensitivity score & Mean age (years) & Age range (years) & Cysts & Cancer & Others \\
\hline All & 86 & $64(74 \%)$ & 51.4 & 24 to 87 & $36(57 \%)$ & $20(31 \%)$ & $8(12 \%)$ \\
Pre/peri-menopausal & 60 & $45(75 \%)$ & 45.5 & 24 to 57 & $28(62 \%)$ & $10(22 \%)$ & $7(16 \%)$ \\
Postmenopausal & 26 & $19(73 \%)$ & 67 & 46 to 87 & $8(42 \%)$ & $10(53 \%)$ & $1(5 \%)$ \\
\hline
\end{tabular}


(a)

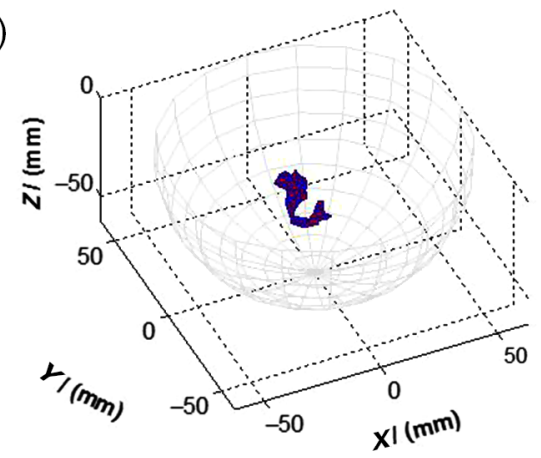

(b)
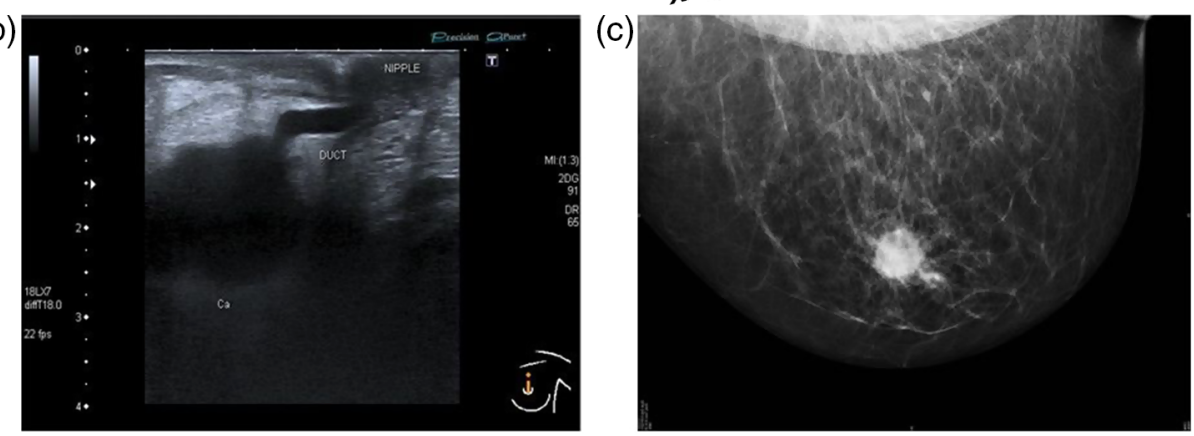

Fig. 2 An example of a MARIA scan compared with a mammogram (MMG) and US. (a) MARIA M4 scan [max focused strength at $(X=3, Y=21, Z=-42 \mathrm{~mm}$ )], (b) US scan, and (c) MMG. Clinical diagnoses: carcinoma $17 \mathrm{~mm}$ and liquid-filled milk duct. Only tumor is visible on mammogram. Both carcinoma and liquid-filled milk duct are visible on MARIA M4 and US scans.

Table 2 Comparison of MARIA M4 detection compared to original radiologist report and a "Blind" review.

\begin{tabular}{lccc}
\hline Characteristic & $\begin{array}{c}\text { MARIA M4 } \\
(n=66)\end{array}$ & $\begin{array}{c}\text { Original } \\
\text { radiologist } \\
\text { report }\end{array}$ & $\begin{array}{c}\text { Single } \\
\text { radiologist } \\
\text { blind review }\end{array}$ \\
\hline Correct detection & $49 / 66(74 \%)$ & $51 / 66(77 \%)$ & $55 / 66(83 \%)$ \\
Lucent (BI-RADS a+b) & $13 / 24(54 \%)$ & $22 / 24(92 \%)$ & $22 / 24(92 \%)$ \\
Dense (BI-RADS c+d) & $36 / 42(86 \%)$ & $29 / 42(69 \%)$ & $33 / 42(79 \%)$ \\
\hline
\end{tabular}

Note: Comparison between MARIA M4 correct detection rate and the original radiographic diagnosis using all available clinical data, both compared with a new review of radiographs alone used by a single radiologist, using the same mix of 66 cases. We note the improved results in dense breasts by the experienced radiologist compared with the original results from a mix of duty radiologists notwithstanding the additional clinical information those radiologists were using. $\mathrm{BI}$ RADS score is based on the American College of Radiologists (fifth edition): (a) the breasts are almost entirely fatty; (b) there are scattered areas of fibroglandular density; (c) the breasts are heterogeneously dense, which may obscure small masses; (d) the breasts are extremely dense, which lowers the sensitivity of MMG.

$86 \%$ (36/42) in MMG dense breasts (BI-RADS c or d), which was a $17 \%$ increase compared to the original clinical review (69\%) and a 7\% increase compared to the blind review (79\%). Examples of MARIA images are given for a grade 3 invasive ductal carcinoma (B5b), where there was good correspondence with the US (U5) and where the MMG was marked as normal (M1) by the original radiologist and an experienced radiologist (Fig. 3). For comparison, a negative example of MARIA is shown in which conventional methods (US and MMG) were successful (Fig. 4).

\section{Discussion}

Although the number of subjects analyzed here is too small to permit extensive statistical comparisons, nevertheless, some trends can be demonstrated. A detection rate of $74 \%$ in all 86 breasts scanned compares very well to the $78 \%$ score in digital MMG reported in the digital mammographic imaging screening trial (DMIST) study. ${ }^{43}$ Further improved results in dense breasts at $86 \%$ compares even more favorably to the DMIST dense breast group at $78 \%$ and these MARIA results in dense breasts is important as women with dense tissue in $75 \%$ or more of the breast have a risk of $\mathrm{BC}$ four to six times as great as the risk among women with little or no dense tissue..$^{13-15,44,45}$ Patients undergoing a MARIA scan reported that the procedure was acceptable and easily managed by those able to lie prone, and still, for about 2 min and particularly appreciated the lack of breast compression. As the incidence of $\mathrm{BC}$ has increased and $\sim 25 \%$ of all deaths due to BC occur in the 40 - to 49 -year-old age group, ${ }^{46,47}$ the MARIA system has potential to provide a major impact in improving BC screening. The MARIA system produces a high-contrast 3-D image of the breast and offers the provision of a safer, more comfortable, and inexpensive breast screening alternative compared to other modalities, which has been shown to be particularly effective at detecting cancer in younger, premenopausal women with dense breasts. MARIA may also overcome some of the challenges posed by trying to optimize the balance between benefit and harm of MMG screening in women of younger age. An improved MARIA M5 system with full CE marking is currently undergoing additional clinical evaluation (approved by Yorkshire \& The Humber and South Yorkshire REC 15/YH/0084, ClinicalTrials.gov NCT02493595). 
(a)

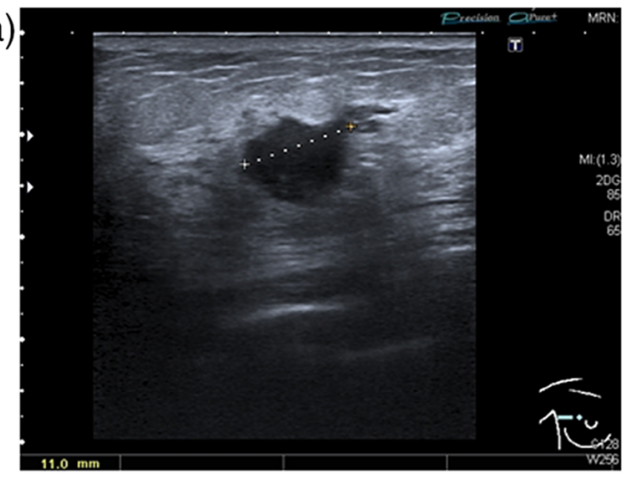

(b)

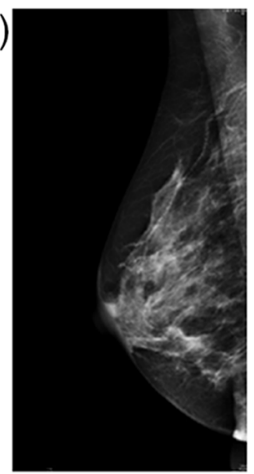

(c)

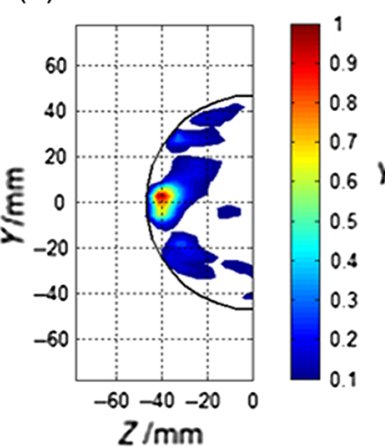

(d)

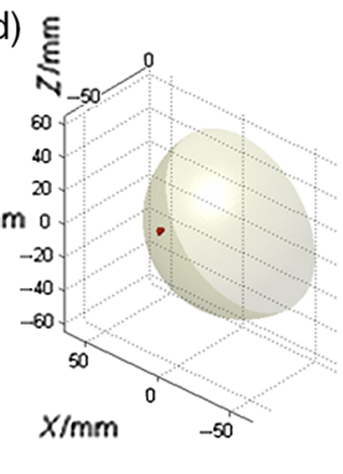

Fig. 3 Comparison of MARIA M4 detection of B5b with US and MMG, BI-RADS c. (a) US scan (U5), (b) MMG marked as normal (M1) by the original and experienced radiologist, (c) lateral view of right breast using MARIA showing area of high dielectric contrast [lateral view of right breast focusing at $X=21 \mathrm{~mm}$, max at $(Y=3, Z=-42 \mathrm{~mm})$ ], and (d) 3-D image of lesion using MARIA [level $=$ $0.8 \max$ at $(X=21, Y=3, Z=-42 \mathrm{~mm})$ value $=1.413 \mathrm{e}-002]$.

(a)

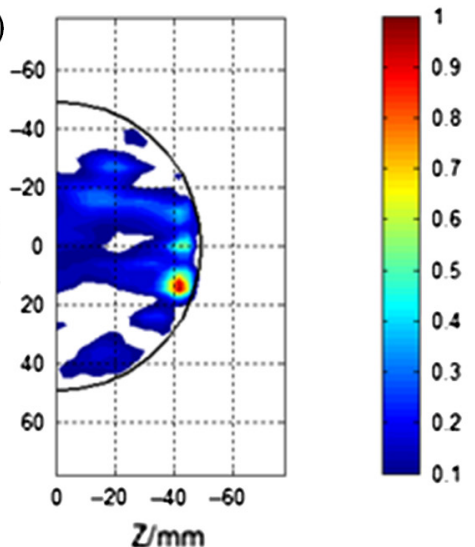

(c)

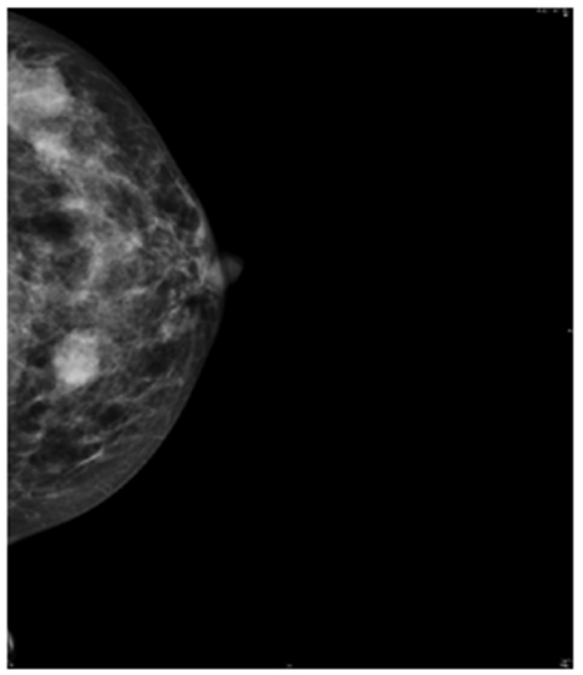

(b)

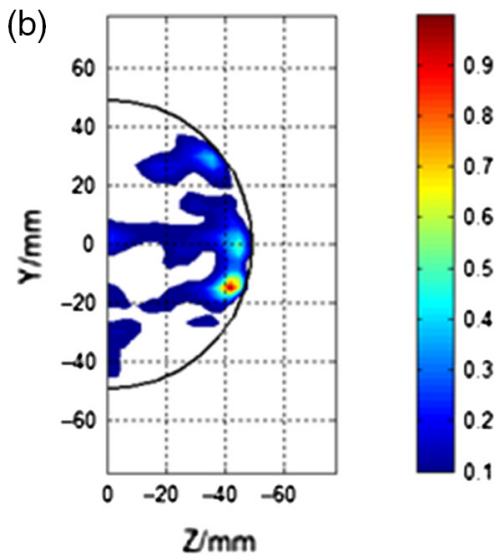

(d)

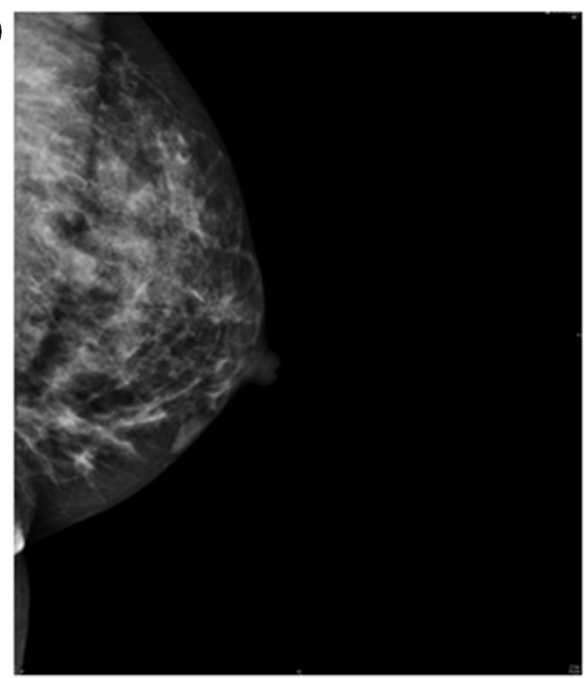

Fig. 4 Comparison of MARIA M4 images where there was a false correspondence with conventional methods (i.e., US and MMG) in detection of a carcinoma. (a) CC view of left breast with MARIA M4 [CC view of right breast focusing at $Y=0.15 \mathrm{~mm}$, max at $(X=15, Z=-42 \mathrm{~mm})$ ], (b) lateral view of left breast with MARIA M4 [lateral view of left breast focusing at $X=15 \mathrm{~mm}$, max at $(Y=0.15$, $Z=-42 \mathrm{~mm}$ )], (c) CC view of left breast with MMG, marked as (M5) in original trial assessment and (M4) by experienced radiologist (LCC), and (d) left medial lateral oblique view by MMG (LMLO).

\section{Conclusion}

Microwave imaging is a rapid, potentially diagnostic technology that is nonionizing, does not involve breast compression, and that has been found to be able to identify regions of significant dielectric contrast, even in dense breasts. This suggests it has value in a routine diagnostic breast care clinic, where $\mathrm{x}$-ray MMG is known to perform suboptimally in dense tissue..$^{13}$ Due to MARIA's completely benign radiation characteristic, the 
technique lends itself to future applications within a younger screening demographic, including women who are deemed to be at a high risk of developing $\mathrm{BC}$.

\section{Acknowledgments}

The authors thank the University Hospitals Bristol and the North Bristol Health Trust for their continuing support. They also would like to thank Dr. Alexandra Valencia; and all the breast care consultants who provided not only advice, but access to patients, and for their assistance and support. This was a joint initiative between the Bristol Health Trusts, the University of Bristol and Micrima, a spinout company of Bristol University, U.K., A.P., and I.C. are the cofounders, and H.W. is an employee of Micrima. The remaining authors have no financial disclosures and had complete, unrestricted access to the study data at all stages of the study. Funding was via research grants to the University of Bristol from Micrima. The derivative of the MARIA 4 designated MARIA 5 has been CE certified as an imaging device, based on the clinical results from MARIA 4 and constructional details, and the group of peer reviewed publications on various elements of the device. A number of patents are in place: P100161 (patent B) and P100163 (patent A). P106281-Methods and apparatus for measuring the internal structure of an object. P109245-Antenna for investigating structure of human or animal. P109246-Methods and apparatus for measuring the contents of a search volume. P119382Methods and apparatus for measuring the internal structure of an object. P120236-Methods, apparatus, and computer-readable medium for assessing fit.

\section{References}

1. F. Bray et al., "Global cancer transitions according to the human development index (2008-2030): a population-based study," Lancet Oncol. 13(8), 790-801 (2012).

2. L. A. Torre et al., "Global cancer statistics, 2012," CA Cancer J. Clin. 65(2), 87-108 (2015).

3. F. Bray et al., "Global estimates of cancer prevalence for 27 sites in the adult population in 2008," Int. J. Cancer 132(5), 1133-1145 (2013).

4. Global Cancer Facts and Figures, 3rd ed., American Cancer Society, Atlanta, Georgia (2015).

5. N. Howlader et al., SEER Cancer Statistics Review (CSR) 1975-2012, National Cancer Institute, Bethesda, Maryland (2016).

6. I. Barco et al., "Comparison of screened and nonscreened breast cancer patients in relation to age: a 2 -institution study," Clin. Breast Cancer 15(6), 482-489 (2015).

7. D. A. Berry et al., "Effect of screening and adjuvant therapy on mortality from breast cancer," N. Engl. J. Med. 353(17), 1784-1792 (2005).

8. R. M. Mann et al., "Breast MRI: EUSOBI recommendations for women's information," Eur. Radiol. 25(12), 3669-3678 (2015).

9. C. Robertson et al., "The clinical effectiveness and cost-effectiveness of different surveillance mammography regimens after the treatment for primary breast cancer: systematic reviews, registry database analyses and economic evaluation," Health Technol. Assess. 15(34), 1-342 (2011).

10. J. E. C. Chubiz et al., "Cost-effectiveness of alternating magnetic resonance imaging and digital mammography screening in BRCA1 and BRCA2 gene mutation carriers," Cancer 119(6), 1266-1276 (2013).

11. C. H. Ahern et al., "Cost-effectiveness of alternative strategies for integrating MRI into breast cancer screening for women at high risk," Br. J. Cancer 111(8), 1542-1551 (2014).

12. J. N. Wolfe, "Breast patterns as an index of risk for developing breast cancer," Am. J. Roentgenol. 126(6), 1130-1137 (1976).

13. N. F. Boyd et al., "Mammographic density and the risk and detection of breast cancer," N. Engl. J. Med. 356(3), 227-236 (2007).

14. G. Ursin et al., "Mammographic density and breast cancer in three ethnic groups," Cancer Epidemiol. Biomarkers Prev. 12(4), 332-338 (2003).
15. N. F. Boyd et al., "Mammographic breast density as an intermediate phenotype for breast cancer," Lancet Oncol. 6(10), 798-808 (2005).

16. C. Kuhl et al., "Prospective multicenter cohort study to refine management recommendations for women at elevated familial risk of breast cancer: the EVA trial," J. Clin. Oncol. 28(9), 1450-1457 (2010).

17. A. W. Preece et al., "Dielectric imaging - an alternative to X-ray mammography?," Br. J. Cancer 64(Supplement XV (P34)), 25 (1991).

18. J. M. Sill and E. C. Fear, "Tissue sensing adaptive radar for breast cancer detection-experimental investigation of simple tumor models," IEEE Trans. Microwave Theory Tech. 53(11), 3312-3319 (2005).

19. E. C. Fear, P. M. Meaney, and M. A. Stuchly, "Microwaves for breast cancer detection?," IEEE Potentials 22(1), 12-18 (2003).

20. E. C. Fear, J. Sill, and M. A. Stuchly, "Experimental feasibility study of confocal microwave imaging for breast tumor detection," IEEE Trans. Microwave Theory Tech. 51(3), 887-892 (2003).

21. X. Li et al., "An overview of ultra-wideband microwave imaging via space-time beamforming for early-stage breast-cancer detection," IEEE Trans. Antennas Propag. 47(1), 19-34 (2005).

22. Q. Li et al., "Direct extraction of tumor response based on ensemble empirical mode decomposition for image reconstruction of early breast cancer detection by UWB," IEEE Trans. Biomed. Circuits Syst. 9(5), 710-724 (2015).

23. M. Klemm et al., "Radar-based breast cancer detection using a hemispherical antenna array-experimental results," IEEE Trans. Antennas Propag. 57(6), 1692-1704 (2009).

24. A. J. Surowiec et al., "Dielectric properties of breast carcinoma and the surrounding tissues," IEEE Trans. Biomed. Eng. 35(4), 257-263 (1988).

25. W. T. Joines et al., "The measured electrical properties of normal and malignant human tissues from 50 to $900 \mathrm{MHz}, "$ Med. Phys. 21(4), 547-550 (1994).

26. A. M. Campbell and D. V. Land, "Dielectric properties of female human breast tissue measured in vitro at $3.2 \mathrm{GHz}$," Phys. Med. Biol. 37(1), 193-210 (1992).

27. M. Lazebnik et al., "A large-scale study of the ultrawideband microwave dielectric properties of normal breast tissue obtained from reduction surgeries," Phys. Med. Biol. 52(10), 2637-2656 (2007).

28. M. Lazebnik et al., "A large-scale study of the ultrawideband microwave dielectric properties of normal, benign and malignant breast tissues obtained from cancer surgeries," Phys. Med. Biol. 52(20), 6093-6115 (2007).

29. C. Gabriel, S. Gabriel, and E. Corthout, "The dielectric properties of biological tissues. 1. Literature survey," Phys. Med. Biol. 41(11), 2231-2249 (1996).

30. S. Gabriel, R. W. Lau, and C. Gabriel, "The dielectric properties of biological tissues. 2. Measurements in the frequency range $10 \mathrm{~Hz}$ to 20 GHz," Phys. Med. Biol. 41(11), 2251-2269 (1996).

31. S. Gabriel, R. W. Lau, and C. Gabriel, "The dielectric properties of biological tissues. 3. Parametric models for the dielectric spectrum of tissues," Phys. Med. Biol. 41(11), 2271-2293 (1996).

32. T. Sugitani et al., "Complex permittivities of breast tumor tissues obtained from cancer surgeries," Appl. Phys. Lett. 107(1), 019902 (2015).

33. R. Benjamin, "Detecting reflective object in reflective medium," U.K. Patent No. GB2313969 (1994).

34. M. Klemm et al., "Improved delay-and-sum beamforming algorithm for breast cancer detection," Int. J. Antennas Propag. 2008, 761402 (2008).

35. M. Klemm et al., "Microwave radar-based differential breast cancer imaging: imaging in homogeneous breast phantoms and low contrast scenarios," IEEE Trans. Antennas Propag. 58(7), 2337-2344 (2010).

36. M. Klemm et al., "Development and testing of a 60-element UWB conformal array for breast cancer imaging," in Proc. of the 5th European Conf. on Antennas and Propagation (EUCAP'11), pp. 3077-3079 (2011).

37. T. Henriksson et al., "Clinical trials of a multistatic UWB radar for breast imaging," in 2011 Loughborough Antennas and Propagation Conf. (LAPC'11), pp. 1-4 (2011).

38. D. Gibbins et al., "A comparison of a wide-slot and a stacked patch antenna for the purpose of breast cancer detection," IEEE Trans. Antennas Propag. 58(3), 665-674 (2010).

39. W. Shao et al., "UWB microwave imaging for breast tumor detection in inhomogeneous tissue," in 27th Annual Int. Conf. of the Engineering in Medicine and Biology Society (EMBS'05), pp. 1496-1499 (2005). 
40. Z. Wang, J. Li, and R. Wu, "Time-delay- and time-reversal-based robust capon beamformers for ultrasound imaging," IEEE Trans. Med. Imaging 24(10), 1308-1322 (2005).

41. J. Leendertz et al., "A liquid phantom medium for microwave breast imaging," in 6th Int. Congress of the European Bioelectromagnetics Association (EBEA), Budapest, Hungary (2003).

42. C. J. D'Orsi et al., ACR BI-RADS® Atlas, Breast Imaging Reporting and Data System, American College of Radiology, Reston, Virginia (2013).

43. E. D. Pisano et al., "Diagnostic performance of digital versus film mammography for breast-cancer screening," N. Engl. J. Med. 353(17), 1773-1783 (2005).

44. C. Byrne et al., "Mammographic features and breast cancer risk: effects with time, age, and menopause status," J. Natl. Cancer Inst. 87(21), 1622-1629 (1995).

45. N. F. Boyd et al., "Quantitative classification of mammographic densities and breast-cancer risk: results from the Canadian national breast screening study," J. Natl. Cancer Inst. 87(9), 670-675 (1995).

46. A. Jemal et al., "Cancer statistics, 2002," CA: Cancer J. Clin. 52(1), 23-47 (2002).

47. S. A. Feig, "Mammographic screening of women aged 40-49 years. benefit, risk, and cost considerations," Cancer 76(10 Suppl), 20972106 (1995).

Alan W. Preece is emeritus professor of medical physics at the University of Bristol, U.K., who previously researched biological and physiological effects of nonionizing radiation on humans. His current work is applied to practical equipment design and the clinical applications of microwave imaging in human subjects for the purpose of identifying and evaluating the imaging possibilities of such microwaves in detection of BC. He is a fellow of the Institute of Physics and Engineering in Medicine.

lan Craddock is a fellow of the IEEE, full Professor at the University of Bristol (UK) and director of the flagship "SPHERE" centre, comprising 100 academics, researchers and graduate students. He has published over 150 papers and serves on the steering board of the University's Health Research Institute. He is also separately employed by Toshiba as managing director of their Telecommunications Laboratory, responsible for a portfolio of communications and loT research.

Mike Shere is a breast clinician, clinical lead of the Bristol Breast Unit at Southmead Hospital, Bristol and clinical lead in North Bristol NHS trust for cancer research. His research interests are: the clinical application of microwave imaging of the breast; high resolution breast ultrasound and breast imaging interventional techniques.

Lyn Jones is a consultant radiologist at Southmead Hospital specialising in vascular and breast radiology and is a fellow of the Royal College of Radiologists and is also trained in surgery.

Helen. L. Winton is a clinical trial manager with Micrima and Research Collaborator with the University of Bristol, UK. Experienced in large multicentre clinical studies, she gained her PhD from Bristol University, investigating the role of immunogenetics in corneal transplant rejection. Currently working alongside breast care clinicians, she is interested in the clinical applications of microwave imaging for breast cancer detection. 